\title{
Nanoindentation of Hydrated Materials and Tissues
}

\author{
Michelle L. Oyen \\ Cambridge University Engineering Department \\ Cambridge, UK \\ mlo29@cam.ac.uk
}

\begin{abstract}
Nanoindentation techniques have recently been adapted for the study of hydrated materials, including biological materials and hydrogels. There are unique challenges associated with handling and testing hydrated samples. For hydrated materials, a poroelastic or poroviscoelastic analysis, which explicitly treats the fluid flow through the porous material, is used to extract material properties from experimental data. Some key results from recent works using nanoindentation to evaluate hydrated materials are reviewed in the context of these challenges. Finally, as these studies represent relatively recent developments in the nanoindentation field, an outlook for the future is presented, in which it is clear that a consensus is emerging for quantitative evaluation of hydrated materials via a modified nanoindentation approach.

keywords: biological tissue, hydrogel, fluid, poroelasticity, nanoindentation
\end{abstract}

\section{Introduction}

For more than three decades, nanoindentation testing has been established as an effective tool for measuring the mechanical properties of materials $[1,2,3,4]$. Nanoindentation is a form of depth-sensing indentation (DSI) testing, in which the full force-displacement-time response is monitored during a contact mechanics experiment, which became popular for measuring material properties at depths of tens to hundreds of nanometers. The majority of early nanoindentation studies focussed on the evaluation of the properties of relatively stiff and hard elastic-plastic engineering materials, such as metals, ceramics, glasses and semiconductors. The most commonly reported material parameters are the (plane strain) elastic modulus $E^{\prime}$ and the hardness $H$-the resistance to (plastic) deformation. A key enabling breakthrough 
that led to the widespread adoption of nanoindentation testing was the development of techniques $[1,2,5]$ for the straightforward deconvolution of $E^{\prime}$ and $H$ from a single nanoindentation test, one typically performed using a sharp Berkovich diamond indenter probe.

The DSI approach was particularly effective for measuring small volumes of material, allowing for quantitative evaluation of thin film mechanical properties $[6,7,8]$, or for mapping properties across inhomogeneous materials at high spatial resolution [9]. Analyses for elastic indentation of a halfspace, based on isotropic elasticity [10] were adapted not just for thin layers and coated systems $[6,7,8]$ but also for elastically anisotropic materials [11]. In addition to nanoindentation experiments, significant effort has been expended on computational modelling of elastic-plastic indentation in particular[12]. This is true of both "forward" simulations, in which the indentation load-displacement response is predicted for a given set of material properties ( $E^{\prime}, H$, hardening law) and of the "inverse" problem, in which attempts are made to extract properties uniquely from load-displacement data.

Following the establishment of robust nanoindentation techniques for characterising engineering materials, the approach was adapted for testing less stiff materials, including bulk polymers [13], polymer coatings [14, 15], and biological materials $[16,17]$. For reasonably stiff materials, including hard and dehydrated biological materials such as bone [18], tooth enamel [19], and plant seed [20], few experimental or analytical adaptations were required, and nanoindentation testing allowed for the spatial mapping of the elastic stiffness of tooth enamel [21] and for the evaluation of elastic anisotropy in bone [22] based on Berkovich indentation with Oliver-Pharr [2] data analysis assuming elastic unloading. However, three sets of challenges have emerged in the context of hydrated materials, and these have prevented the establishment of a standardized testing routine for nanoindentation measurements of material properties. First, there are inherent challenges due to the fact that the samples are hydrated and the instrumentation was designed for testing dry samples. Second, many-although not all-hydrated materials are significantly less stiff than typical non-hydrated engineering materials. Third, there is no consensus on the appropriate data analysis for interpreting data obtained from tests on hydrated samples. Thus, these three factors taken together have limited the development of routine nanoindentation testing of hydrated materials and tissues, and each will be described here in turn. We will conclude with an outlook for the future of nanoindentation of hydrated 
materials.

\section{Hydrated Materials}

Two types of hydrated materials will be considered here: biological tissues and hydrogels. "Biological tissues" is a broad term encompassing both plant and animal tissues, in which the fundamental make-up of a tissue is biological cells plus extracellular matrix materials $[23,24]$. The categories of biological tissues can be further sub-divided into "hard" and "soft" tissues, where the words hard and soft do not imply anything with respect to hardness or plastic deformation. Hard tissues, such as bone [18, 22], calcified cartilage [25], enamel[19, 21] and dentin [26] in teeth, or nacre in sea shell [27], contain significant biomineral content, such as calcium phosphate or calcium carbonate. Soft tissues such as cartilage [28] and artery [29] are non-mineralised in their healthy state. Mammalian tissues have been studied using nanoindentation largely in the context of biomedical applications, informing the community about the disease process in conditions such as osteoporosis [30], and evaluating the effect of drugs or treatments for disease [31]. Other nonclinical research involves elucidating basic structure-properties relationships in natural materials more generally, including both mammalian and non-mammalian animal tissues and plant tissues.

Hydrogels are hydrophilic polymeric materials in which the polymer chains are allowed to swell in water. The polymer can be synthetic or natural in source and the polymer cross linking can be chemical or physical [42]. There are two components to the total water content, water that is tightly bound to the polymer network and water that is free to move through small pores within the polymer network (Figure 1) [32]. Because of their large water contents, hydrogels are quite biocompatible, and have been used as materials for soft contact lenses [33], coatings on medical devices [34], and wound dressings [35]. It is anticipated that the use of hydrogels in medicine will continue to expand for applications such as drug delivery applications, diagnostics, and in tissue engineering [36]. Hydrogels have also increasingly been used in basic-science biological studies investigating cell-material interactions [37], including those in which gel mechanical properties are varied systematically in order to study mechanical influences on stem cell differentiation [38, 39]. The mechanical behavior of hydrogels has long been recognized as fundamentally important but fundamentally lacking due to the large volume occupied by water [40]. As such, recent studies have considered hydrogel composites 
[41] to try and improve on the baseline material properties of hydrogels while maintaining their biocompatibility. Nanoindentation has been used to characterise the material properties of hydrogels, which can be difficult to "grip" for traditional mechanical testing due to their compliance and hydrated state [42]

\section{Testing Hydrated Samples}

For nanoindentation experiments on hydrated samples, the state of material hydration must be maintained during the test. There are at least three basic ways that this has been done. Samples can be fully hydrated in fluid and then placed into the chamber of the nanoindenter, such that testing takes place quickly before the fluid evaporates. A time-frame for testing can be established by weighing samples at multiple time-points on removal from a fluid bath to check for evaporation. This approach demonstrated no loss of mass for polyacrylamide hydrogels in the first hour in air [43], establishing an acceptable time-frame for testing of just under one hour. Samples of both hydrogels and soft biological tissue have been tested when surrounded by a hydrating foam layer, with weight-loss studies demonstrating that the samples were maintained in a hydrated state identical to fully submerged samples over the course of eight hours [29]. Samples can also be tested while fully submerged in fluid [44], although this can cause challenges as capillary forces have been shown to interfere with sample surface detection [45]. A detailed and quantitative exploration of the capillary forces has been performed as a function of indenter geometry [46]. Special indenter probes with longer than typical shafts are often used for fluid-immersed samples, to increase the distance between the fluid and the electronics of the DSI transducer.

A maintenance of hydration state allows for comparisons between nanoindentation results for hydrated versus dehydrated samples of the same material. Bone is approximately $20 \%$ water by volume, but a number of studies have demonstrated that dry bone is stiffer than hydrated bone with as much as an order of magnitude difference in elastic modulus [18, 22, 25, 47, 48, $49,50])$. A systematic study of the influence of hydration on the same bone samples showed that the differences can in part be explained by differences in probe geometry and data analysis method between different studies [51] (Figure 2). However, when wet and dry samples were compared using the same probe geometry and data analysis method, plane strain modulus values for wet bone were $60-80 \%$ of those observed for the same dry bone [51]. Even 
more striking, reported hardness values for wet bone were only 30-35\% of those for dry bone, illustrating that the total deformations are much greater when the bone is hydrated. This hardness difference was consistent with the observation that total indentation displacement was greater in wet than in dry tooth dentin, giving smaller hardness values and less normalised energy dissipation during the indentation cycle [26]. Most of the literature on bone and tooth nanoindentation considers dry samples, which means the absolute values reported for properties cannot be considered quantitative. Similarly, if even more dramatic, observations have been made for hydrogels, where hydrogels with originally $80 \%$ water were three orders of magnitude stiffer when dehydrated in either air or by immersion in ethanol [43].

There have been interesting observed differences from testing samples immersed in different types of fluid environments. Differences have been observed between the hydrated nanoindentation response of bone when tested in water and in physiological saline solution, with stiffer responses observed for saline than for water $[48,47]$. In the same study, the effects of a range of polar solvents and combinations of solvents were considered, and it was observed that bone properties changed dramatically with the fluid environment. The effects of fluid polarity on bone paled in comparison to those observed in collagen, where elastic modulus increased by orders of magnitude with decreasing values of the solvent dielectric constant, suggesting that soft tissues are far more susceptible to fluid composition than bone [52, 53]. Because of the potential for rapid degradation of a biological sample ex vivo, antibiotics have been added to the hydrating fluid [54]. For polymeric materials, it is possible to test in a detergent solution instead of in water, which has been shown to result in data that are not influenced by the fluid or adhesion between the probe tip and the sample [45]. However, this is not an option for biological materials for which it would not be physiological to test in a detergent solution. Questions have arisen as to the ideal conditions for storage of samples for later testing. The process of formalin fixation significantly increases the stiffness of fibrous soft biological tissues and is often avoided, while a freezing-thawing cycle has less obvious effects on the tissue properties [55] and is common (and practical) practice. Most nanoindentation studies in a fluid environment have also been conducted at room temperature and not at body temperatures, further indicating that the properties measured are indicative but not necessarily quantitatively physiological. 


\section{Testing Compliant Samples}

Although mineralised tissues such as bone have stiffnesses that are comparable to many engineering materials, hydrated soft tissues and hydrogels are much more compliant, with elastic modulus values in the $\mathrm{kPa}$ to $\mathrm{MPa}$ range instead of in the GPa range. This introduces several challenges in nanoindentation testing, particularly when commercial instrumentation is utilized. There are three primary challenges to overcome in this context, to do with measurement resolution, with surface detection, and intrinsic time-dependent deformation in the material.

First, the testing of compliant samples works in the opposite force-displacement regime from stiff materials, with small forces at relatively large displacements compared with large forces at relatively small displacements [24]. For this reason, the probe geometry is often changed from that of a sharp, pyramidal Berkovich tip [2] to a spherical [50] or flat-punch [56] tip. This increases the contact area and therefore the contact stiffness, allowing for measurements within the working load and displacement ranges and resolutions of the instrument [24].

Regardless of probe geometry, there can be difficulties with the unambiguous detection of the sample surface when the sample is compliant. Whether the surface detection is by a force change or by a stiffness change, a compliant sample may undergo significant deformation before the instrumentation sets the "zero point". This has been shown to lead to significant overestimation of the elastic modulus in compliant samples [57]. The challenges with surface detection are further complicated by the presence of fluid on the sample surface (i.e., if the specimen is submerged) as the fluid surface tension results in apparent forces before the probe contacts the sample [45]. To overcome some of these challenges, feedback control has been utilised, such that a displacement controlled testing mode can be utilised instead of the load controlled mode typically found in commercial nanoindenters [43]. The test can be initiated when the probe is slightly above the sample but in contact with the fluid, and the zero-point correction can be applied to the data post-hoc.

Compliant materials frequently exhibit time-dependent mechanical behaviour, which further introduces wrinkles into the experimental protocol. The time-dependent deformation in compliant samples requires further consideration in the context of nanoindentation experiments. The first issue to be addressed is the form of the load function for the test. There are two fre- 
quently used control modes, load-control or displacement-control, and experiments can either be quasistatic or oscillatory [24]. Quasistatic experiments are similar to typical loading profiles used for testing elastic-plastic materials, with a constant loading- or displacing-rate for loading and unloading, with or without a hold at peak load or displacement. A hold segment at the peak point is advantageous when testing time-dependent materials, because this segment is sensitive to the creep or load-relaxation occurring in the sample. For oscillatory testing, there has been a recent rise in commercially available nanoindentation equipment set-ups for contact-based measurement, in which stiffness measurements are obtained as a function of frequency [56, 58, 59]. These measurements are often of little utility, as the raw dependence of stiffness on frequency is not converted to true material property data.

Complicating matters, since the development of commercial nanoindenter instruments, the correction for thermal drift has included measuring the baseline change in displacement at a small, fixed force with the indenter tip resting on the sample surface, either prior to or after performing the nanoindentation test. However, this cannot be easily disambiguated from the intrinsic creep displacement in the sample, or, on unloading, the creep-recovery. It is therefore important to disable the thermal drift correction procedure when testing time-dependent materials using commercial nanoindenters. Finally, the time-dependent deformation must be considered explicitly in the analysis of nanoindentation data for hydrated materials, and this will be considered in detail next.

\section{Poroelastic Analysis of Nanoindentation Data}

The time-dependence demonstrated by hydrated materials has been described using a poroelastic model [60,61], which describes a material's constitutive response by the flow of a fluid through a porous elastic solid. The stresses and strains in the elastic skeleton are coupled to changes in the internal pore pressure and the fluid volume; following the application of a load to a poroelastic material, the rise in pore pressure gives rise to time-dependent motion of the unbound fluid (Figure 1). When equilibrium is reached, the total fluid volume contained within the solid skeleton will have changed. The material properties of the elastic porous skeleton, the shear modulus $G$ and the (drained) Poisson's ratio $\nu$ dictate the elastic behaviour. There are three additional material constants required for a full poroelastic specification, to describe the fluid-solid coupling and the fluid flow. Different sets 
of the five constants can be utilized depending on the assumptions made, such as whether the undrained Poisson's ratio is taken as $0.5[60,61]$. The flow behaviour is dictated by the Darcy permeability, $\kappa$, which relates the fluid flux to the pressure gradient, and which is the most commonly reported poroelastic parameter in addition to the solid skeleton elastic properties $G$ and $\nu$. The Darcy (hydraulic) permeability can be considered as the ratio of an intrinsic permeability $k$ to the fluid viscosity $\eta$ such that $k=\kappa \eta$. The intrinsic permeability with units of length ${ }^{2}$, is a direct indicator of pore size and illustrates that there is more resistance to fluid motion when the pore size-and the intrinsic permeability-is small. The intrinsic permeability can be estimated from first principles for a material with known solid fraction and characteristic dimensions [62].

The poroelastic constitutive laws, like those for thermoelasticity, are coupled and as such there are very few mechanical problems for which poroelastic problems can be solved analytically. In particular, for indentation geometries, computational approaches have been used [43, 49, 63, 64, 65, 66, 67]. Individual experimental data sets can be inversely fit using finite element analysis [43], but this is computationally expensive and can be tedious for large data sets. More commonly, experimental nanoindentation data can be fit to a database of forward model responses with interpolation between simulations $[65,67]$ or fit to a master curve $[63,64]$.

An interesting physical consequence of the association of permeability with pore size in the sample is that length-scale effects arise when testing hydrated poroelastic materials. This is a key distinguishing feature between poroelastic time-dependent deformation and viscoelastic time-dependent deformation, which occurs in solid polymers and has no inherent length-scale effects. There is a length scale associated with poroelasticity, $L$, and this length scale is dictated by the experiment, as it represents the path length of fluid travel. For an indentation test, this length scale is taken as the radius of contact, whereas for a compression test it is related to the sample size. For a poroelastic material, the length of time from application of force to equilibrium has an effective time constant $\tau=L^{2} / G \kappa$. Thus, both the intrinsic material properties, the modulus $G$ and permeability $\kappa$, and the extrinsic length scale $L$ dictate the load relaxation behaviour in poroelastic materials. This introduces an important reason for working to overcome limitations or challenges associated with performing nanoindentation tests on compliant, hydrated materials: the time to equilibrium is smaller when the contact area is smaller. 
For a polyacrylamide hydrogel, indented with a spherical probe at a macroscopic length scale, relaxation occurs only over a time-scale of hours, but with much smaller scale spherical nanoindentation, the time-scale is minutes [43]. This is illustrated in Figure 3, which shows finite element simulations for the nanoindentation and microindentation experiments on polyacrylamide gels using the experimental set-ups and material properties obtained in [43]. In the experimental time-frame of a few minutes, the microindentation test appears to show no relaxation while the nanoindentation test does. However, when the full responses are normalised and compared, it is clear that the relaxation had not yet commenced for the microindentation experiment. It is crucial to recall in comparing these experiments and simulations that the material properties are unchanged, only the length scale of the experiment has changed. Testing hydrated poroelastic materials is thus more efficient for smaller contacts, and thus ideally suited for nanoindentation.

Table 1 shows a summary of poroelastic material properties of a range of hydrated biological samples and hydrogels as measured by indentation and nanoindentation testing. (In cases where the intrinsic permeability was reported rather than the hydraulic permeability, conversion was performed assuming a value of $\eta=1 \mathrm{mPa}$ s for water.) In several of these studies, indenter probes of vastly different radii were used to explicitly examine lengthscale effects $[44,69,74]$; when size effects were found, nanoindentation (i.e., smaller radius) values are reported here. As can be seen, the elastic modulus of the hydrogels and soft tissue are orders of magnitude smaller than those of bone. The permeability values generally trend upwards with decreasing material stiffness, which is consistent with basic scaling laws; from polymer physics, elastic modulus increases with the square of gel solid-fraction and permeability varies as solid-fraction to the -1.5 [42]. The large number of available results, most reported from the last five years, demonstrate that indentation measurement of the poroelastic properties of hydrated materials is a quickly growing endeavor.

An additional consequence of the distinction between viscoelasticity and poroelasticity-with its associated length scale-is that these two effects can be separated uniquely for materials exhibiting both behaviours simultaneously. This requires multiple experiments, and can be performed in several ways $[68,71]$. For indentation testing, the easiest way to manipulate the lengthscale is to change the radius of a spherical indenter probe and indent to the same depth, resulting in systematic variation in the contact radius [68]. The set of responses can be fit to a poroelastic-viscoelastic model as a group 
Table 1: Poroelastic properties from indentation tests on hydrated samples

\begin{tabular}{|l|l|l|}
\hline Material & Shear Modulus, $G$ & Permeability, $\kappa\left[\mathbf{m}^{4}(\mathbf{N s})^{-1}\right]$ \\
\hline Bone [69] & 0.6 to $1.2 \mathrm{GPa}$ & $1 \times 10^{-21}$ to $5 \times 10^{-20}$ \\
\hline Bone [44] & $0.5 \mathrm{GPa}$ & $6.5 \times 10^{-20}$ \\
\hline Bone [67] & $0.47 \mathrm{GPa}$ & $6.5 \times 10^{-20}$ \\
\hline Bone [49] & $0.43 \mathrm{GPa}$ & $4.65 \times 10^{-21}$ \\
\hline Cartilage [72] & $740 \mathrm{kPa}$ & $6.6 \times 10^{-17}$ \\
\hline Cartilage [44] & $236 \mathrm{kPa}$ & $6.6 \times 10^{-17}$ \\
\hline Polyacrylamide gels [43] & 298 to $365 \mathrm{kPa}$ & $6.25 \times 10^{-17}$ to $1.30 \times 10^{-16}$ \\
\hline Polyacrylamide gels [74] & 6.2 to $6.8 \mathrm{kPa}$ & $4.2 \times 10^{-15}$ to $5.8 \times 10^{-15}$ \\
\hline Polyethylene glycol gel [64] & $100 \mathrm{kPa}$ & $5.4 \times 10^{-16}$ \\
\hline Gelatin gel [70] & $27 \mathrm{kPa}$ & $9.1 \times 10^{-13}$ \\
\hline Gelatin gel [67] & $24.2 \mathrm{kPa}$ & $1.1 \times 10^{-12}$ \\
\hline Gelatin gels [73] & 3 to $30 \mathrm{kPa}$ & $4 \times 10^{-14}$ to $2 \times 10^{-13}$ \\
\hline Agar gel [68] & $98 \mathrm{kPa}$ & $1.1 \times 10^{-13}$ \\
\hline Agar gels [73] & 10 to $100 \mathrm{kPa}$ & $2 \times 10^{-13}$ to $2 \times 10^{-12}$ \\
\hline Alginate gel [63] & $27.9 \mathrm{kPa}$ & $3.6 \times 10^{-13}$ \\
\hline
\end{tabular}

for extraction of material parameters. The overall force is found to be the product of the viscoelastic and poroelastic contributions, indicating that the two mechanisms act relatively independently. This independence makes sense since one mechanism is dictated by polymer chain dissipation (i.e., the porous solid skeleton) and the other by fluid flow.

\section{Conclusion and Outlook}

This overview has considered the nanoindentation of hydrated biological tissues and hydrogels. In both classes of materials, water is an intrinsic part of the material, and the properties that are measured in the dry material are nothing like those observed in the hydrated state. As such, it is important to develop methods for keeping the samples wet during nanoindentation testing, whether by testing samples quickly after removing them from fluid or by testing them in a hydrated or submerged fluid environment. These experiments are reasonably straightforward using typical commercial nanoindentation instrumentation for mineralised hard tissue such as bone, tooth tissues, or marine creatures, in which the overall response is dominated by the stiff calcium phosphate or calcium carbonate phase. It is more 
complicated when testing hydrated soft tissues such as cartilage or hydrogels such as polyacrylamide. These materials are $75 \%$ water or more, and as such have material elastic modulus values many orders of magnitude smaller than those for which commercial instrumentation was developed.

The analysis of nanoindentation data for hydrated materials is necessarily different from that of elastic-plastic engineering materials such as metals or ceramics. A poroelastic mechanical framework explicitly takes into account the fluid phase and its transport through the porous solid skeleton made of polymer or biopolymer molecules. The mechanical response of poroelastic materials to applied loads is therefore time-dependent, and this timedependence has an intrinsic length scale associated with the pore size of the material. Poroelastic experiments are therefore faster to execute when the experimental length-scale is smaller, meaning that nanoindentation is ideally suited for routine material property measurements in hydrated materials.

In order for nanoindentation of hydrated materials to become commonplace, several things are required from the community. With stiff and hard elastic-plastic materials, there is a uniform consensus about the instrumentation, design of experiment, and analysis of data. A lot of this relates back to the seminal 1992 paper of Oliver and Pharr [2] and the subsequent incorporation of the Oliver-Pharr methodology into commercial nanoindentation systems. A Berkovich tip ships with the instruments by default, and the reported properties are the elastic modulus and hardness as measured from the unloading portion of the load-displacement curve.

With hydrated materials, no such consensus exists, although particularly for compliant soft tissues and hydrogels, one may be slowly emerging (Table 2). Instrumentation ranges widely, including custom devices [63, 64], repurposed universal test frames $[43,44,39]$ and commercial nanoindenters when the materials are sufficiently stiff $[43,44,65]$. Most studies use spherical indenter probes and poroelastic data analysis, although this is all done off-line from the mechanical testing instrument, utilising custom codes and algorithms. A large gap exists between this emerging consensus for hydrated compliant materials and common practices for stiff biocomposites, whicheven when samples are hydrated-still tend to largely follow the consensus associated with the stiffer materials and Oliver-Pharr approach, perhaps because it almost works when the fluid content of the material is small. However, in order for the soft materials approach to take hold, it will likely require at least one manufacturer of scientific instruments to both design an instrument suited for these material types and to include software for facile data 
Table 2: Experimental nanoindenation techniques, adapted from [24]

\begin{tabular}{|c|c|c|}
\hline Variable & Stiff material & Hydrated material \\
\hline Probe tip geometry & Berkovich & Spherical \\
\hline Control mode & Load-control & Displacement-control \\
\hline $\begin{array}{l}\text { Thermal drift correc- } \\
\text { tion }\end{array}$ & $\mathrm{On}$ & Off \\
\hline Data analyzed & Unloading & $\begin{array}{l}\text { Holding (load relax- } \\
\text { ation) }\end{array}$ \\
\hline Data analysis & $\begin{array}{ll}\text { Oliver-Pharr } & {[2]} \\
\text { (elastic-plastic) } & \end{array}$ & Poroelastic $[63,67]$ \\
\hline Parameters reported & $\begin{array}{l}\text { Plain strain modulus } \\
E^{\prime}, \text { Hardness } H\end{array}$ & $\begin{array}{l}\text { Shear modulus } G \text {, Per- } \\
\text { meabilty } \kappa \text { or } k \text {, often } \\
\text { Poisson's ratio } \nu\end{array}$ \\
\hline
\end{tabular}

analysis. Without a more turn-key way of executing tests on soft tissues and hydrogels, it is unlikely that nanoindentation of hydrated materials will really take off. And without a simple mechanism for performing routine mechanical testing on soft hydrated materials, it is likely that the development of mechanically robust hydrogels for biomedical applications will be considerably slowed.

\section{Acknowledgements}

The author acknowledges Robert F. Cook for helpful feedback on an early draft of this work, and Daniel GT Strange for performing the finite element analysis in Figure 3 in the course of his PhD studies. 


\section{References}

\section{References}

[1] Doerner MF and Nix WD, 15. A method for interpreting the data from depth-sensing indentation instruments J. Mater. Res. 1, 601 (1986).

[2] Oliver WC and Pharr GM, Improved technique for determining hardness and elastic modulus using load and displacement sensing indentation experiments, J. Mater. Res. 7 1564-83 (1992).

[3] Oliver WC and Pharr GM, Measurement of hardness and elastic modulus by instrumented indentation: Advances in understanding and refinements to methodology, J. Mater. Res. 19 3-20 (2004).

[4] Cook RF, Probing the Nanoscale, Science 328 183-4 (2010).

[5] G.M. Pharr, W.C. Oliver, and F.R. Brotzen, On the generality of the relationship among contact stiffness, contact area, and elastic modulus during indentation, J. Mater. Res. 7, 613 (1992).

[6] King RB, Elastic analysis of some punch problems for a layered medium, Int J Solids Struct 23, 1657 (1987).

[7] Rar A, Song H, Pharr GM, Assessment of New Relation for the Elastic Compliance of a Film-Substrate System. Mat. Res. Soc. Symp. Proc. 695 L10.10.1-6 (2002).

[8] Saha R and Nix WD, Effects of the substrate on the determination of thin film mechanical properties by nanoindentation, Acta Materialia 2002, 50, pp 23-28.

[9] Constantinides G, Ravi Chandran KS, Ulm F-J, and van Vliet KJ, Grid indentation analysis of composite microstructure and mechanics: Principles and validation, Materials Science and Engineering a-Structural Materials Properties Microstructure and Processing, 430, 189-202 (2006).

[10] I.N. Sneddon, The relation between load and penetration in the axisymmetric Boussinesq problem for a punch of arbitrary profile, Int. J. Engng. Sci. 3, 47 (1965). 
[11] Vlassak JJ, Ciavarella M, Barber JR, Wang X, The indentation modulus of elastically anisotropic materials for indenters of arbitrary shape. J. Mech. Phys. Solids 51 (2003) 1701-21.

[12] Cheng Y-T and Cheng C-M, Scaling, dimensional analysis, and indentation measurements. Mater Sci Eng Reports 44 (2004) 91-149.

[13] Briscoe BJ, Fiori L, Pelillo E, Nano-indentation of polymeric surfaces. J. Phys. D: Appl. Phys. 31 2395-2405 (1998).

[14] Oyen ML, Cook RF, Moody NR, Emerson JA, Indentation Responses of Time-Dependent Films on Stiff Substrates. J. Mater. Res. 19 2487-97 (2004).

[15] Zhang CY, Zhang YW, Zeng KY, Extracting the mechanical properties of a viscoelastic polymeric film on a hard elastic substrate. J. Mater. Res. 2004, 19(10):3053-61.

[16] Ebenstein D and Pruitt L, Nanoindentation of biological materials. Nano Today 1 26-33 (2006).

[17] Oyen ML (ed.) Handbook of Nanoindentation with Biological Applications. Singapore: Pan Stanford Publishing (2010).

[18] Rho J-Y, Tsui TY, Pharr GM, Elastic properties of human cortical and trabecular lamellar bone measured by nanoindentation. Biomaterials $\mathbf{1 8}$ 1325-1330 (1997).

[19] Marshall GW, Balooch M, Gallagher RR, Gansky SA, Marshall SJ, Mechanical properties of the dentinoenamel junction: AFM studies of nanohardness, elastic modulus, and fracture, Journal of Biomedical Materials Research, Vol. 54, 8795 (2001)

[20] Lucas PW, Cook RF, Lowrey TK, How Baby Plants Avoid Getting Hurt and Blossom into Adulthood: The Story of a Tropical Seed, in Mechanics of Biological and Bio-Inspired Materials, Ed. C. Viney, K. Katti, C. Hellmich, and U. Wegst, MRS Symposium Proceedings Vol. 975 (2007) DOI: 10.1557/PROC-975-0975-DD08-07.

[21] Cuy JL, Mann AB, Livi KJ, Teaford MF, and Weihs TP, Nanoindentation mapping of the mechanical properties of human molar tooth enamel. Arch. Oral. Biol. 47 281-91 (2002). 
[22] Swadener, J.G., Rho, J.-Y. and Pharr, G.M. Effects of anisotropy on elastic moduli measured by nanoindentation in human tibial cortical bone. J. Biomed. Mater. Res. 57 108-112 (2001).

[23] B. Alberts, A. Johnson, J. Lewis, M. Raff, K. Roberts, and P. Walter: Molecular Biology of the Cell, 4th ed. [Garland Science (Taylor and Francis), New York, 2002].

[24] Oyen ML, Nanoindentation of Biological and Biomimetic Materials, Experimental Techniques 37 (2013) $73-87$.

[25] Ferguson VL, Bushby AJ, and Boyde A, Nanomechanical properties and mineral concentration in articular calcified cartilage and subchondral bone. J. Anat.203 191-202 (2003).

[26] Bertassoni LE, Swain MV, Influence of hydration on nanoindentation induced energy expenditure of dentin. J. Biomech 45 (2012) 1679-83.

[27] Bruet BJF, Qi HJ, Boyce MC, Panas R, Tai K, Frick L, Ortiz C, Nanoscale morphology and indentation of individual nacre tablets from the gastropod mollusc Trochus niloticus, J. Mater. Res. 20 (2005) 240019.

[28] Ebenstein DM, Kuo A, Rodrigo JJ, Reddi AH, Ries M, Pruitt L, A nanoindentation technique for functional evaluation of cartilage repair tissue. J. Mater. Res. 19 (2004) 273-81.

[29] Ebenstein DM and Pruitt LA, Nanoindentation of soft hydrated materials for application to vascular tissues. J. Biomed. Mater. Res. 69A (2004) 222-32.

[30] Guo XE and Goldstein SA, Vertebral trabecular bone microscopic tissue elastic modulus and hardness do not change in ovariectomized rats. J. Orthop Res. 18 (2000) 333-6.

[31] Amanata N, He LH, Swain MV, Little DG. The effect of zoledronic acid on the intrinsic material properties of healing bone: an indentation study. Med. Eng. Phys. 30 (2008) 843-7.

[32] Hoffman AS, Hydrogels for biomedical applications, Adv. Drug Delivery Rev. 2002, 43, 3-12. 
[33] Wichterle O and Lim D, Hydrophilic Gels for Biological Use, Nature 1960, 185, 117 - 118.

[34] Yang SH, Lee YS, Lin FH, Yang JM, Chitosan/poly(vinyl alcohol) blending hydrogel coating improves the surface characteristics of segmented polyurethane urethral catheters. J Biomed Mater Res B Appl Biomater. 2007, 83 304-13.

[35] Corkhill PH, Hamilton CJ, Tighe BJ, Synthetic hydrogels. VI. Hydrogel composites as wound dressings and implant materials. Biomaterials 1989, 10, 3-10.

[36] Peppas NA, Hilt JZ, Khademhosseini A, Langer R. Hydrogels in Biology and Medicine: From Molecular Principles to Bionanotechnology, Adv. Mater. 2006, 18, 1345-60.

[37] Brandl F, Sommer F, Goepferich A, Biomaterials 2007, 28, 134-46.

[38] Engler AJ, Sen S, Sweeney HL, Discher DE, Rational design of hydrogels for tissue engineering: impact of physical factors on cell behavior. Cell 2006, 126, 677-89.

[39] Trappmann B, Gautrot JE, Connelly JT, Strange DGT, Li Y, Oyen ML, Cohen Stuart MA, Boehm H, Li B, Vogel V, Spatz JP, Watt FM, Huck WTS Extracellular-matrix tethering regulates stem-cell fate. Nature Materials 11 (2012) 642-9.

[40] Baroli B, Hydrogels for tissue engineering and delivery of tissue-inducing substances, J. Pharmaceutical Sci. 2007, 96, 2197-2223.

[41] Butcher AL*, Offeddu GS*, Oyen ML, Nanofibrous hydrogel composites as mechanically robust tissue engineering scaffolds. Trends in Biotechnology 32 (2014) 564-70. (* Joint first authors.)

[42] Oyen ML, Mechanical Characterization of Hydrogel Materials, International Materials Reviews 59 (2014) 44 - 59.

[43] Galli M, Comley KSC, Shean TAV, Oyen ML, Viscoelastic and Poroelastic Mechanical Characterization of Hydrated Gels. J. Mater. Res. 24 973-9 (2009). 
[44] Oyen ML, Shean TAVS, Strange DGT, Galli M, Size Effects in Indentation of Hydrated Biological Tissues, Journal of Materials Research 27 (2012) $245-55$.

[45] Ebenstein DM, Nano-JKR force curve method overcomes challenges of surface detection and adhesion for nanoindentation of a compliant polymer in air and water, J. Mater. Res. 26 (2011) 1026-35.

[46] Chen SH and Soh AK, The capillary force in micro- and nanoindentation with different indenter shapes. Int. J. Solids Structures 45 (2008) 3122-37.

[47] Bembey AK, Oyen ML, Bushby AJ, Boyde A, Viscoelastic Properties of Bone as a Function of Hydration State Determined by Nanoindentation. Phil. Mag. 86 5691-703 (2006).

[48] Bembey AK, Bushby AJ, Boyde A, Ferguson VL, Oyen ML, Hydration Effects on the Micro-Mechanical Properties of Bone. J. Mater. Res. 21 1962-8 (2006).

[49] Oyen ML, Poroelastic Nanoindentation Responses of Hydrated Bone. J. Mater. Res. 23 1307-14 (2008).

[50] Bushby AJ, Ferguson VL, and Boyde A: Nanoindentation of bone: Comparison of specimens tested in liquid and embedded in polymethylmethacrylate. J. Mater. Res. 19 249-59 (2004).

[51] Rodriguez N, Oyen ML, Shefelbine SJ, Insight into Differences in Nanoindentation Properties of Bone, J. Mech. Behav. Biomed. Mater. 18 (2013) 90 - 99.

[52] Ntim MM, Bembey AK, Ferguson VL, Bushby AJ, Hydration effects on the viscoelastic properties of collagen. Materials Research Society Symposium Proceedings 898E, 0898-L05-02, 1-6 (2006)

[53] Galli M and Oyen ML, Bone Composite Mechanics Related to Collagen Hydration State, Proceedings of the IUTAM Symposium on Cellular, Molecular and Tissue Mechanics, (held at Woods Hole, MA, June 2008). Published (2010) 269-76. 
[54] Hoffler CE, Moore KE, Kozloff K, Zysset PK, Brown MB, Goldstein SA, Heterogeneity of bone lamellar-level elastic moduli. Bone 26 603609 (2000).

[55] Ebenstein DM, Coughlin D, Chapman J, Li C, Pruitt LA, Nanomechanical properties of calcification, fibrous tissue, and hematoma from atherosclerotic plaques. J. Biomed. Mater. Res. 91A (2009) 1028-37.

[56] EG Herbert, WC Oliver, A Lumsdaine, GM Pharr Measuring the constitutive behavior of viscoelastic solids in the time and frequency domain using flat punch nanoindentation Journal of Materials Research 24 (2009) 626-637.

[57] Kaufman JD and Klapperich CM, Surface detection errors cause overestimation of the modulus in nanoindentation on soft materials. J. Mech. Behav. Biomed. Mater. 2 312-317 (2009).

[58] Nayar VT, Weiland JD, Nelson CS, Hodge AM, Elastic and viscoelastic characterization of agar. J. Mech. Behav. Biomed. Mater. 7 (2012) 60-8.

[59] Franke O, Goeken M, Meyers MA, Durst K, Hodge AM, Dynamic nanoindentation articular porcine cartilage, Mater Sci Eng C 31 (2011) 789-95.

[60] H.W. Wang, Theory of Linear Poroelasticity with Applications to Geomechanics and Hydrogeology, Princeton, NJ : Princeton University Press (2000).

[61] Detournay E, Cheng AH-D, Fundamentals of Poroelasticity, In: Fairhurst, C. (Ed.), Comprehensive Rock Engineering: Principles, Practice and Projects, Vol. II, Analysis and Design Method, Pergamon, 113171 (1993)

[62] Jackson, G.W., James, D.F., 1986. The permeability of fibrous porousmedia. Can. J. Chem. Eng. 64 (3), 364374.

[63] Hu Y, Zhao X, Vlassak JJ, Suo Z. Using indentation to characterize the poroelasticity of gels. Applied Phys. Lett. 96121904 (2010).

[64] Chan EP, Hu Y, Johnson PM, Suo Z, Stafford CM, Spherical indentation testing of poroelastic relaxations in thin hydrogel layers. Soft Matter 8 (2012) 1492-8. 
[65] Gupta S, Lin J, Ashby P, Pruitt L, A fiber reinforced poroelastic model of nanoindentation of porcine costal cartilage: A combined experimental and finite element approach. J. Mech. Behav. Biomed. Mater. 2 326-38 (2009).

[66] Galli M and Oyen ML, Spherical Indentation of a Finite Poroelastic Coating. Appl. Phys. Lett. 93031911 (2008).

[67] Galli M and Oyen ML, Fast Identification of Poroelastic Parameters from Indentation Tests. CMES (Comp. Modeling Eng. Sci.) 48 241-68 (2009).

[68] Strange DGT, Fletcher TL, Tonsomboon K, Brawn H, Zhao X, Oyen ML, Separating poroviscoelastic deformation mechanisms in hydrogels. Applied Physics Letters 102, (2013) 031913.

[69] Rodriguez N, Oyen ML, Shefelbine SJ, Age-related changes in mouse bone permeability, J. Biomechanics 47 (2014) 1110 - 6.

[70] Galli M, Fornasiere E, Cugnoni J and Oyen ML, Poroviscoelastic Characterization of Particle-Reinforced Gelatin Gels Using Indentation and Homogenization. J. Mech. Behav. Biomed. Mater. 4 (2011) 610 - 7.

[71] Wang Q-M, Mohan AC, Oyen ML, Zhao X-H, Separating viscoelasticity and poroelasticity of gels with different length and time scales. Acta Mechanica Sinica 30 (2014) 20 - 27.

[72] Miller GJ and Morgan EF, Use of microindentation to characterize the mechanical properties of articular cartilage: comparison of biphasic material properties across length scales Osteoarthritis Cartilage 18 (2010) $1051-7$.

[73] Strange DGT and Oyen ML, Composite Hydrogels for Nucleus Pulposus Tissue Engineering. J. Mech. Behav. Biomed. Mater. 11 (2012) 16-26.

[74] Kalcioglu ZI, Mahmoodian R, Hu Y, Suo Z, Van Vliet KJ, From macroto microscale poroelastic characterization of polymeric hydrogels via indentation, Soft Matter 8 (2012) 3393-8. 


\section{Figure Captions}

Figure 1: A schematic illustration of the cross-section a hydrated poroelastic material. There is bound water in the porous solid skeleton and free water in the pore space. On application of a force, the free water moves in response to pressure gradients; the pores are interconnected and accessible to the external fluid environment.

Figure 2: Plane strain elastic modulus and wet/dry modulus ratio data plotted for four different nanoindentation experiments on the same bone samples, varying the indenter probe and data analysis method [51]. The value of the plane strain modulus varies significantly from experiment to experiment, but there is always a substantial difference between values for samples tested wet versus dry.

Figure 3: Finite element simulations of nanoindentation (nI) and microindentation $(\mu \mathrm{I})$ experiments on polyacrylamide hydrogels based on the experiments and material property values in [43]. The responses are normalised and plotted together, illustrating that in the larger microindentation experiment, within the experimental time-frame of a few minutes, the load-relaxation has not yet begun. 
Figure 1

Click here to download high resolution image

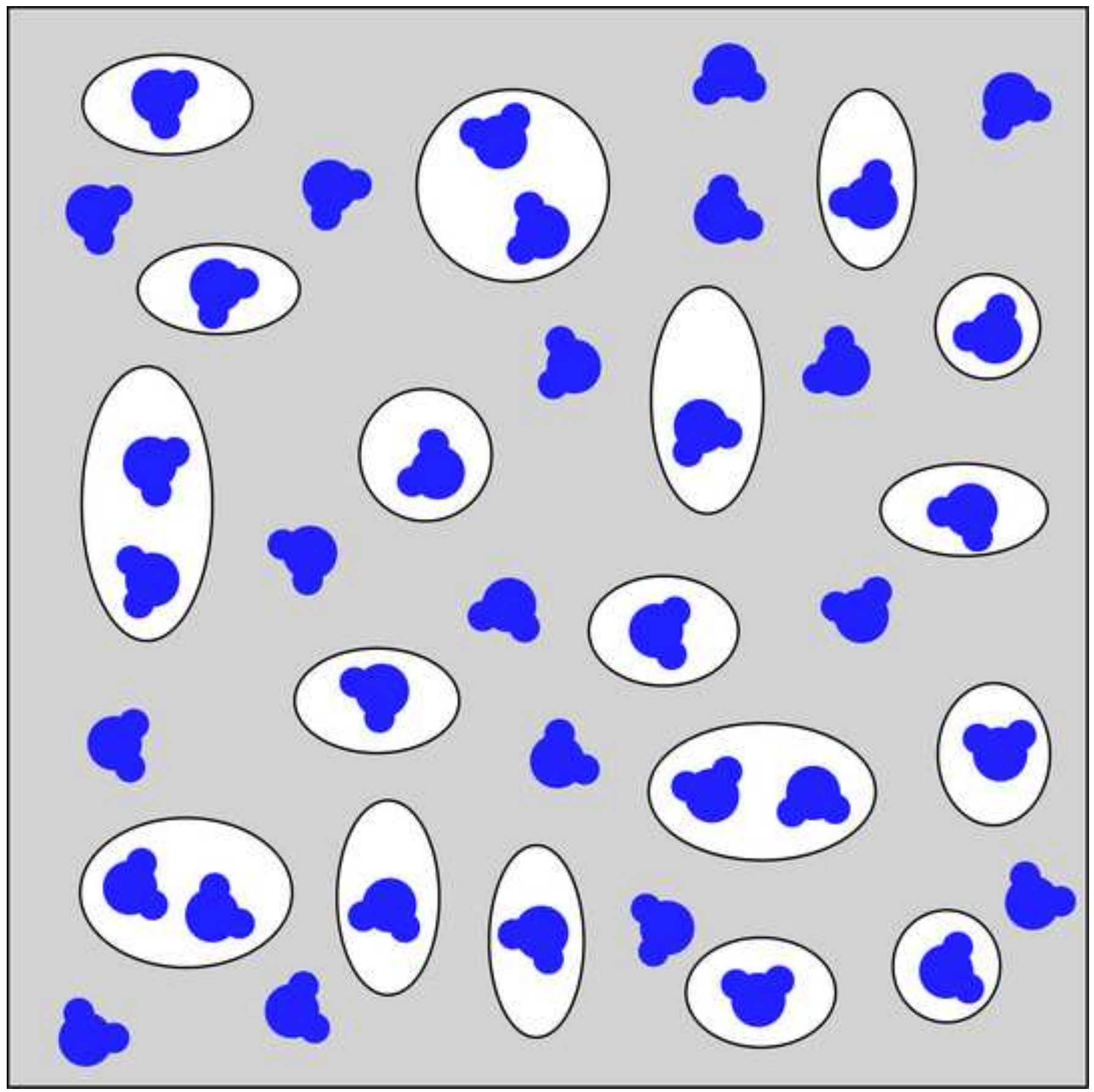




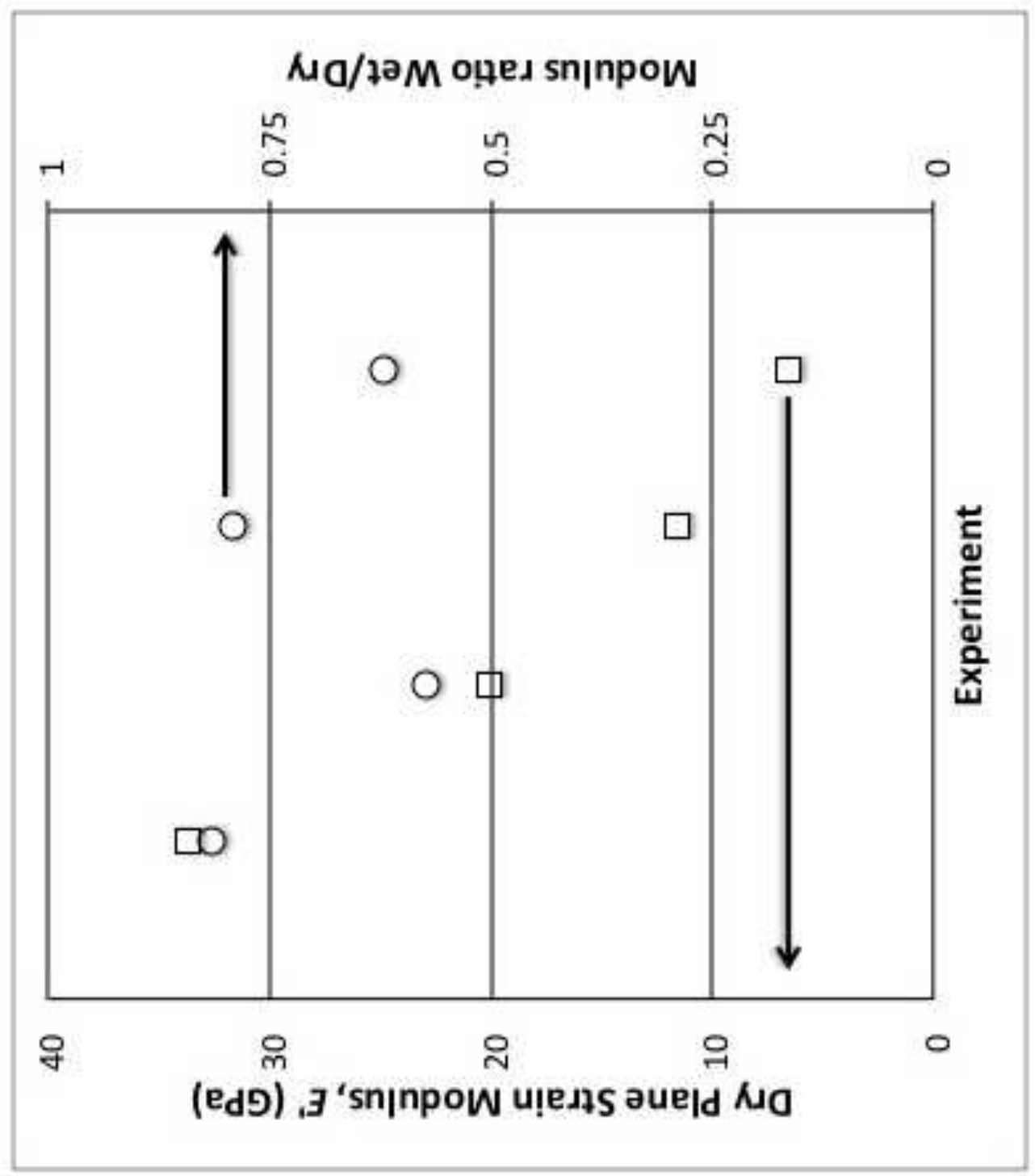




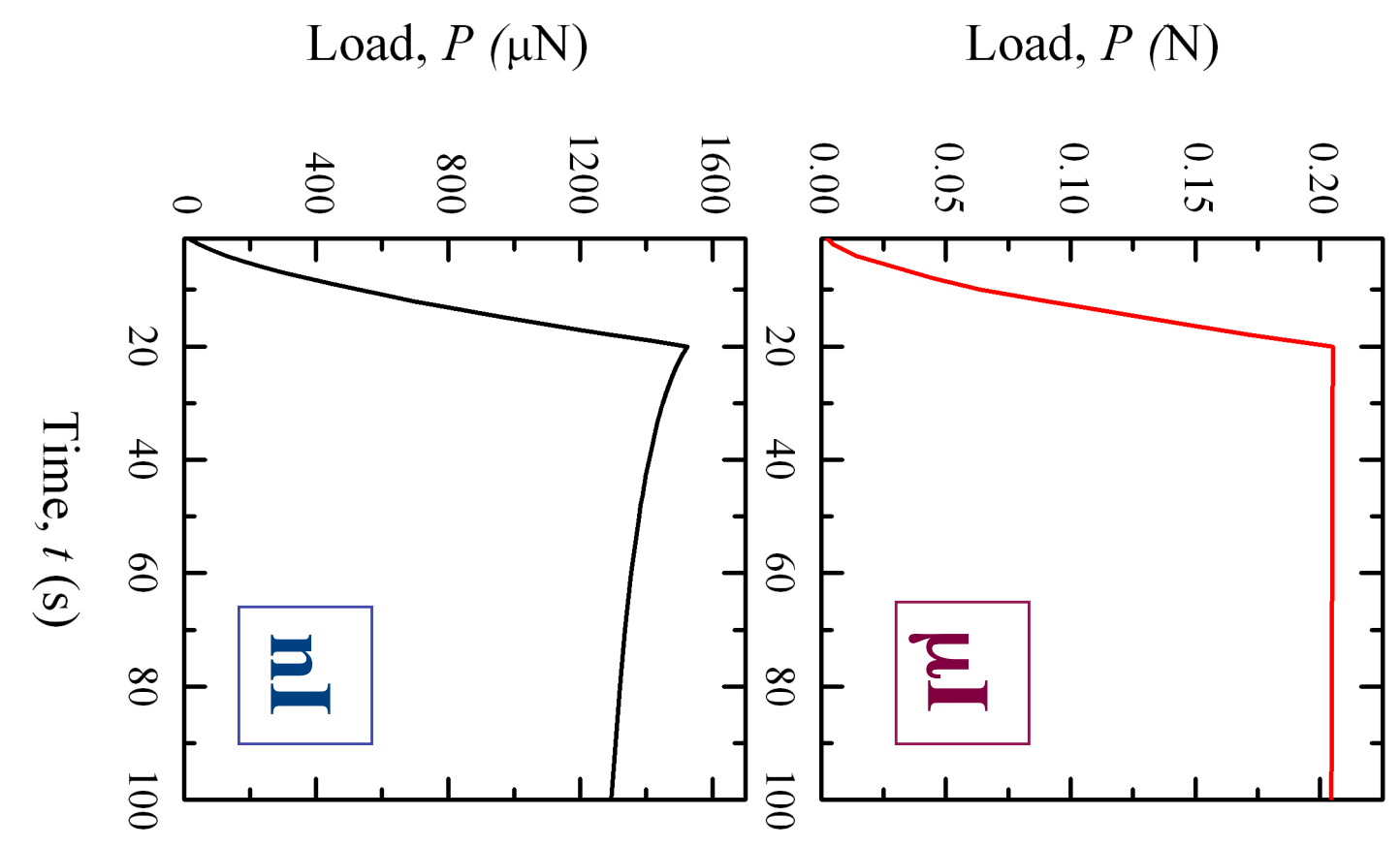

Normalized Load, $P(t) / P_{\infty}$

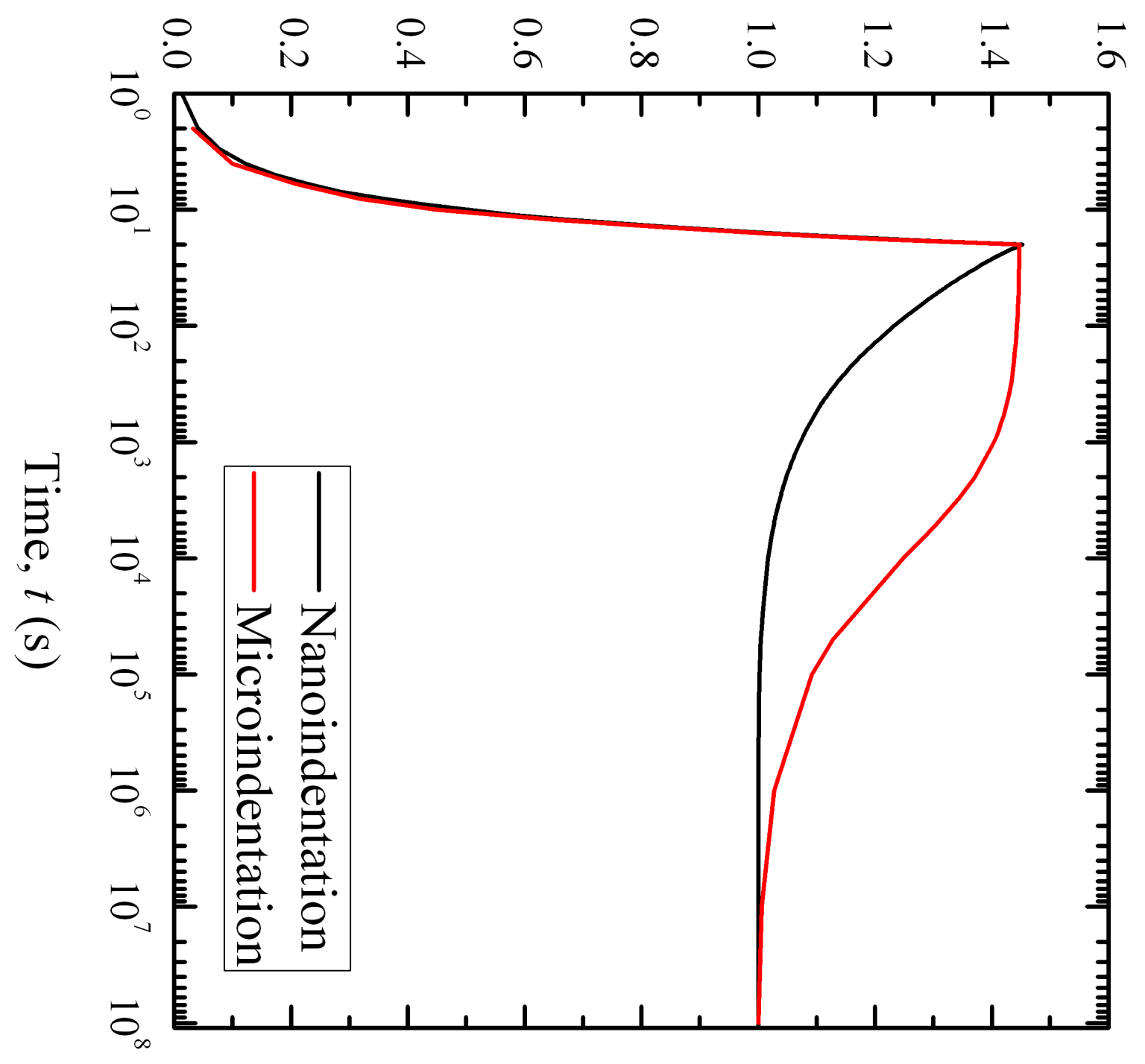

